\title{
Optimizing outcomes in EGFR mutation-positive NSCLC: which tyrosine kinase inhibitor and when?
}

\author{
Nicolas Girard*,1,2 \\ ${ }^{1}$ Thoracic Oncology, Université de Lyon, Université Claude Bernard Lyon 1, Lyon, 69622, France \\ ${ }^{2}$ Thoracic Surgery, Institut Curie, Institut du Thorax Curie-Montsouris, Paris, 75248, France \\ *Author for correspondence: nicolas.girard2@curie.fr
}

\begin{abstract}
Despite the efficacy of standard-of-care EGFR tyrosine kinase inhibitors (TKIs), erlotinib, gefitinib and afatinib, in EGFR mutation-positive non-small-cell lung cancer, resistance develops, most commonly due to the T790M mutation. Osimertinib showed clinical activity in the treatment of T790M-positive disease following progression on a first-line TKI, and is approved in this setting. Recently, osimertinib improved efficacy versus first-generation TKIs (erlotinib and gefitinib) in the first-line setting. Multiple factors can influence first-line treatment decisions, including subsequent therapy options, presence of brain metastases and tolerability, all of which should be considered in the long-term treatment plan. Further research into treatment sequencing is also needed, to optimize outcomes in EGFR mutation-positive non-small-cell lung cancer.
\end{abstract}

First draft submitted: 17 November 2017; Accepted for publication: 15 December 2017; Published online: 16 January 2018

Keywords: afatinib • dacomitinib $\bullet$ EGFR $\bullet$ erlotinib $\bullet$ gefitinib $\bullet$ non-small-cell lung cancer $\bullet$ osimertinib $\bullet$ resistance - treatment sequencing $\bullet$ T790M

Somatic mutations of the EGFR gene are found in up to $50 \%$ of Asian patients and $10-15 \%$ of Caucasian patients with lung adenocarcinoma [1]. Tumors with these mutations become dependent on EGFR signaling, enabling molecularly targeted inhibition of this pathway with tyrosine kinase inhibitors (TKIs) to be used as treatment [2]. Erlotinib and gefitinib were the first EGFR TKIs developed; they reversibly and competitively inhibit the tyrosine kinase domain of EGFR (Figure 1) [3]. Known as the first-generation EGFR TKIs, they rapidly became standard of care first-line treatment for EGFR mutation-positive non-small-cell lung cancer (NSCLC), and molecular testing for $E G F R$ mutations in patients with lung adenocarcinoma is now recommended as part of baseline workup [4]. The hypothesis that broader inhibition of the EGFR family could improve outcomes led to the development of second-generation ERBB family blockers, afatinib and dacomitinib. These agents irreversibly bind to EGFR, ERBB2 and ERBB4 and block transphosphorylation of ERBB3 to inhibit all ERBB family signaling (Figure 1) [5]. Currently, erlotinib, gefitinib and afatinib are standard of care for the first-line treatment of $E G F R$ mutation-positive NSCLC [4].

Despite their proven efficacy, resistance to these treatments inevitably develops. The most common mechanism of resistance is acquisition of another EGFR mutation, EGFR T790M (around $50-70 \%$ of cases) [6,7]. The most recent additions to the EGFR TKI family - the third-generation EGFR TKIs - add to the targeting of EGFR-activating mutations, with specific activity on EGFR T790M (Figure 1). One such agent, osimertinib, demonstrated striking efficacy in patients with EGFR T790M-positive disease that had progressed on a first- or second-generation EGFR TKI and is approved in this setting [7-9]. These data highlight the importance of molecular testing throughout treatment to understand tumor evolution and enable the use of targeted therapies.

Most recently, osimertinib has been assessed as a first-line treatment for EGFR mutation-positive NSCLC compared with a first-generation EGFR TKI (erlotinib or gefitinib), demonstrating a significant improvement in median progression-free survival (PFS), and thus positioning it as another potential first-line treatment option [10]. Consequently, physicians now have a number of options available and need to consider how best to use these

Future Medicine 


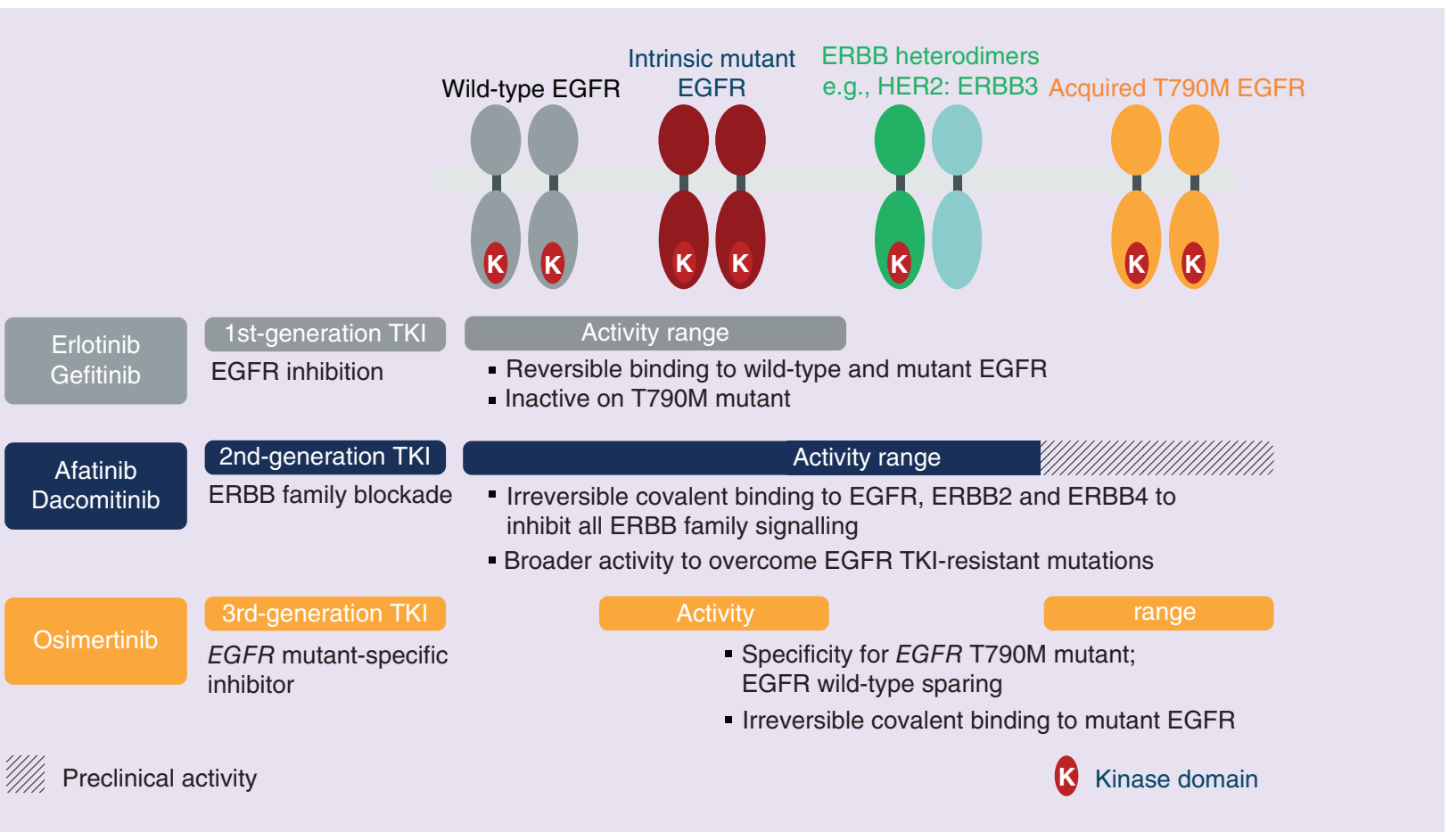

Figure 1. Overview of activity range of first-, second- and third-generation EGFR tyrosine kinase inhibitors.

TKI: Tyrosine kinase inhibitor.

agents to provide the best chance of long-term survival, while also considering the impact on quality of life, which is of particular importance to patients with advanced cancer. Based on the molecular evolution of lung tumors, sequencing of EGFR TKIs may be key to obtaining the best outcome. In this review, we consider the data supporting the use of the available EGFR TKIs and key considerations for selection of first-line treatment.

\section{First- \& second-generation EGFR TKIs}

The first-generation EGFR TKIs, erlotinib and gefitinib, demonstrated superior efficacy to platinum-based chemotherapy in several Phase III trials (OPTIMAL [11], EURTAC [12] and ENSURE [13] for erlotinib, and IPASS [subgroup of EGFR mutation-positive NSCLC] [14], WJTOG3405 [15] and NEJ002 [16] for gefitinib [Table 1]). In these trials, erlotinib and gefitinib were associated with median PFS of 9.2-13.1 months compared with 4.6-6.3 months among patients receiving platinum-based chemotherapy. However, none of the trials were able to show an overall survival (OS) improvement with the first-generation TKIs, which was thought to be related to crossover from chemotherapy to an EGFR TKI upon progression [13,17-20]. Overall, the EGFR TKIs were tolerable, with key adverse events (AEs) including class-related gastrointestinal and skin toxicities (discussed in further detail later).

The second-generation ERBB family blocker, afatinib, has also demonstrated significantly improved PFS versus platinum-based chemotherapy in patients with EGFR mutation-positive NSCLC in two large, randomized Phase III trials (Table 1). In LUX-Lung 3, in patients with common mutations (Del19/L858R), median PFS was 13.6 months for afatinib versus 6.9 months with cisplatin-pemetrexed [25]. These results were confirmed in the LUX-Lung 6 trial, which was conducted in Asia [27]. Subgroup analysis showed that the results were consistent irrespective of age, gender and race. While PFS generally favored afatinib irrespective of mutation type, benefit was most pronounced among those with Del19-positive disease (Table 1) [25,27]. This has also been observed for the first-generation EGFR TKIs [31,32]. LUX-Lung 3 and 6 both also included prespecified analyses of patient-reported outcomes, whereby afatinib was associated with longer time to deterioration and improvements in lung cancer-related symptoms, such as cough, dyspnea and pain $[25,27,33]$. Moreover, in contrast with the first-generation EGFR TKIs, afatinib was associated with an OS benefit in both of these trials. Among patients with Del19-positive NSCLC, median OS was significantly longer in patients receiving afatinib than those receiving chemotherapy (LUX-Lung 3: median, 33.3 vs 


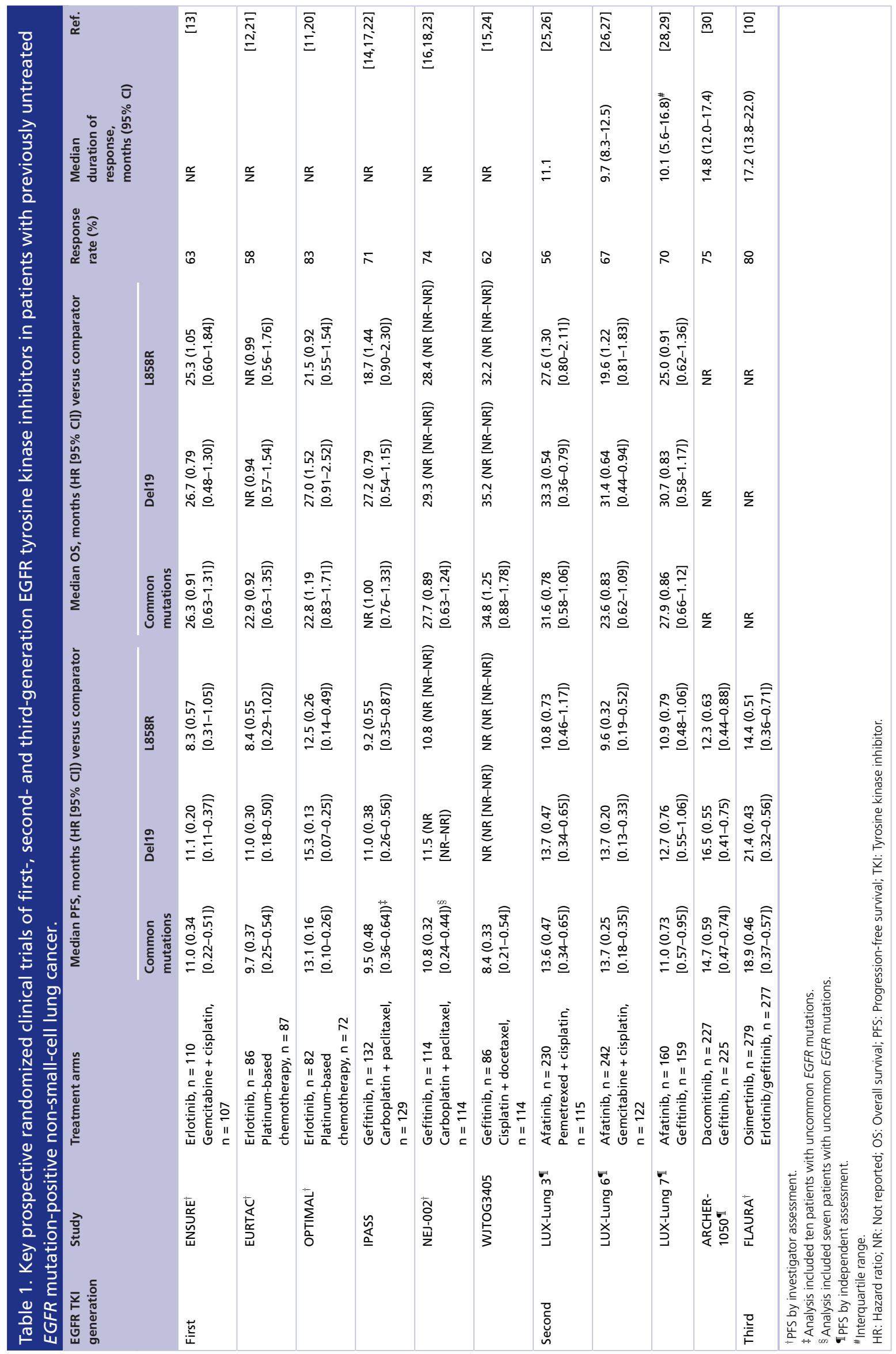


21.1 months, $\mathrm{p}=0.0015$; LUX-Lung 6: median, 31.4 vs 18.4 months, $\mathrm{p}=0.023$ [Table 1]) [26]. It was hypothesized that the observed OS benefit may be related to broad inhibition of the ERBB family by afatinib compared with reversible inhibition of EGFR only by first-generation EGFR TKIs. In addition, given the availability of firstgeneration TKIs at the time those trials were conducted, a significant proportion of patients did receive additional lines of TKIs after the study treatment, which may have impacted OS.

However, until recently, there were no head-to-head trials comparing first- and second-generation EGFR TKIs to substantiate the hypothesis that second-generation EGFR TKIs were more effective. The exploratory Phase IIB LUX-Lung 7 trial, which compared first-line afatinib with gefitinib in EGFR mutation-positive (Del19 or L858R) NSCLC, was the first prospective, randomized trial to compare first- and second-generation TKIs [28]. Patients received treatment until disease progression, or beyond if deemed beneficial by the investigator. Given the exploratory nature of the trial, no formal hypothesis was specified. Three coprimary end points were selected: PFS by independent central review, OS and time to treatment failure (TTF). TTF, which was defined as the time from randomization until treatment discontinuation for any reason, including progression, AEs or death, was included to reflect clinical practice where many patients receive treatment beyond radiological progression in the absence of clinical deterioration. In LUX-Lung 7, afatinib significantly prolonged PFS (median, 11.0 vs 10.9 months; hazard ratio [HR]: $0.73 ; \mathrm{p}=0.017$ ) and TTF (median, 13.7 vs 11.5 months; HR: $0.73 ; \mathrm{p}=0.0073$ ) versus gefitinib. A significantly higher proportion of patients achieved an objective response with afatinib (70\%) versus gefitinib (56\%; Table 1) [28]. Median OS was not significantly different between treatment arms (27.9 months with afatinib vs 24.5 months with gefitinib; HR: 0.86; $\mathrm{p}=0.2580$ ). However, LUX-Lung 7 was not powered for OS, as the sample size was based on controlling the width of the CI for the HR of PFS [29]. As in LUX-Lung 3 and LUX-Lung 6 , the efficacy findings were generally consistent across patient subgroups of gender, age and ethnicity. Furthermore, the efficacy benefits of afatinib over gefitinib were not affected by EGFR mutation type [28,29].

More recently, another second-generation EGFR TKI, dacomitinib, has been assessed in the first-line setting versus gefitinib in the Phase III ARCHER 1050 trial [34]. This study enrolled patients with EGFR mutation-positive (Del19 or L858R) NSCLC, but excluded patients with brain metastases. Dacomitinib significantly prolonged PFS compared with gefitinib (14.7 vs 9.2 months; HR: 0.59; $\mathrm{p}<0.0001$; Table 1). While the proportion of patients who achieved an objective response was similar between the two groups ( $75 \%$ in the dacomitinib group and $70 \%$ in the gefitinib group), duration of response was longer with dacomitinib than gefitinib (HR: $0.55 ; \mathrm{p}<0.0001$ ). OS data were not mature at the time of data cutoff. Although cross trial comparisons of LUX-Lung 7 and ARCHER 1050 should be cautioned, especially given differences among patient populations, the cumulative evidence suggests that second-generation EGFR TKIs may be more effective than first-generation EGFR TKIs.

\section{Third-generation EGFR TKIs in patients with acquired resistance}

Despite the efficacy of first- and second-generation EGFR TKIs, resistance inevitably develops, usually within 10-14 months after starting treatment. The predominant mechanism of resistance appears to be acquisition of EGFR T790M, occurring in 50-70\% of patients [6,7]. The third-generation EGFR TKIs were developed to target this mechanism, showing high potency for T790M and EGFR TKI-sensitizing mutations, although the pharmacological potency for the common Del19/L858R mutations is lower with osimertinib than secondgeneration EGFR TKIs $[35,36]$. A number of third-generation TKIs are in development, but osimertinib is the only agent approved in Europe and the USA for treatment of advanced EGFR T790M mutation-positive NSCLC, based on impressive data from the Phase I/II AURA, Phase II AURA2 and Phase III AURA3 studies. AURA enrolled patients with advanced NSCLC that had progressed after treatment with EGFR TKIs. In the Phase I part, the response rate for osimertinib was 61\%, with a median PFS of 9.6 months among patients who had centrally confirmed EGFR T790M [8]. These results were confirmed in the Phase II AURA extension, in patients with EGFR TKI-pretreated EGFR T790M-positive disease, where the response rate was reported as $62 \%$ and median PFS was 12.3 months [7]. Similarly, in the AURA2 study in patients who had progression on previous EGFR TKI therapy, the response rate was $70 \%$ [37]. Pooled OS analysis from AURA2 and the AURA extension showed median OS of 26.8 months from initiation of osimertinib in patients with T790M-positive NSCLC that had progressed after a first- or second-line EGFR TKI [38]. Osimertinib was subsequently assessed in the randomized Phase III AURA3 trial in patients with T790M-positive advanced NSCLC who had disease progression after first-line EGFR TKI therapy. Patients were randomized to osimertinib or platinum-based therapy plus pemetrexed. Osimertinib significantly prolonged investigator-assessed PFS versus chemotherapy (10.1 vs 4.4 months; HR: 0.30; 95\% CI: 
(A)

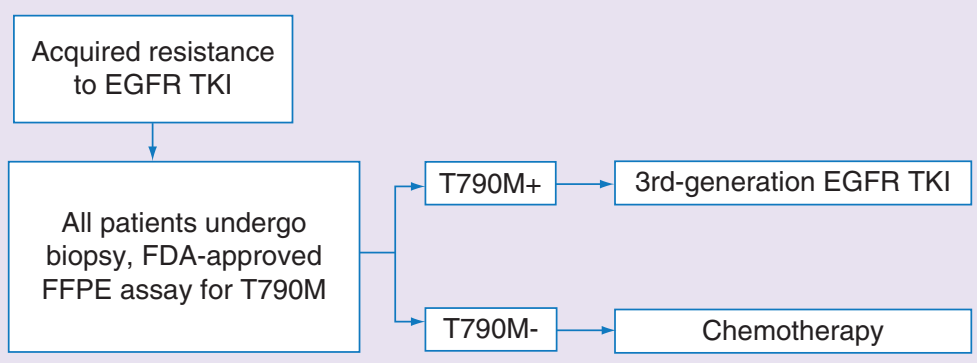

(B)

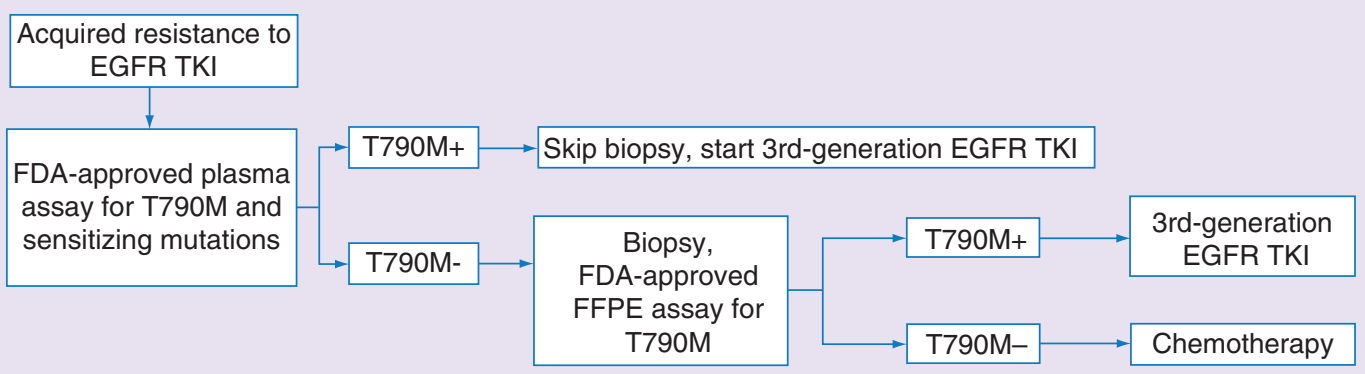

Figure 2. Testing algorithms for detection of T790M. Conventional paradigm (A) and an alternate proposal with the incorporation of plasma-based assays (B).

FFPE: Formalin-fixed, paraffin-embedded; TKI: Tyrosine kinase inhibitor.

Reprinted with permission (c) (2016) American Society of Clinical Oncology. All rights reserved [40].

$0.23-0.41 ; \mathrm{p}<0.001)$ and was associated with a significantly higher response rate (71 vs $31 \%$; odds ratio [OR]: 5.39; 95\% CI: 3.47-8.48; $\mathrm{p}<0.001)$. OS data have not been reported [9].

With the emergence of third-generation EGFR TKIs, this is the first time a biopsy at progression has been needed to guide subsequent therapy. However, biopsy may not always be feasible due to accessibility, and even if it is, the procedure is not without risk. In addition, scheduling the biopsy and performing testing can ultimately delay subsequent therapy. Biopsies are also associated with a failure rate of $5-10 \%$, despite availability of sufficient tissue, and they may not reflect differences in tumors across sites [39]. As a result, there is increased interest in noninvasive genotyping by analysis of cell-free plasma DNA. A post hoc analysis of AURA showed that sensitivity of plasma genotyping for EGFR T790M was $70 \%$ in patients who were T790M-positive on central tumor genotyping [40]. Moreover, outcomes were similar in patients with T790M-positive disease that was detected in plasma or based on tumor biopsy [40]. Some patients had positive T790M on tissue but not on liquid biopsy, related to the lower sensitivity of plasma analyses. Interestingly, some patients had positive T790M on liquid biopsies that was not observed in the tumor tissue, a situation which may reflect spatial heterogeneity, with possible impact on the efficacy of osimertinib. As such, the authors suggested that plasma genotyping could be performed as an initial step to detect T790M mutations, and that patients who were T790M-positive by plasma could proceed with third-generation TKI therapy without biopsy; however, those with T790M-negative results in plasma should have a tumor biopsy to confirm T790M-negativity/positivity and determine subsequent therapy (Figure 2). Further analysis of methods for detection of EGFR mutations (Del19, L858R and T790M) in plasma was performed using data from the AURA3 trial, which enrolled only patients with confirmed T790M mutations [41]. Three plasma assays - allele-specific PCR, droplet digital PCR and next-generation sequencing - were used, with droplet digital PCR and next-generation sequencing showing higher sensitivity for detection of T790M compared with allele-specific-PCR [41]. 


\section{Third-generation EGFR TKIs as first-line treatment}

Osimertinib has recently been evaluated in a first-line setting in the randomized Phase III trial, FLAURA [10]. In this study, patients with common (Del19/L858R) EGFR mutations were randomized to osimertinib or a firstgeneration EGFR TKI, whereby the specific EGFR TKI (erlotinib or gefitinib) was chosen by the site as the sole comparator prior to site initiation. The primary end point - PFS by investigator assessment - was significantly improved in the osimertinib arm versus the erlotinib/gefitinib arm (median, 18.9 vs 10.2 months; HR: 0.46; 95\% CI: 0.37-0.57; $\mathrm{p}<0.0001$; Table 1). PFS benefit was consistent across subgroups, including race, age, gender and presence of stable CNS metastases at baseline. The response rate was similar between treatment arms (80\% with osimertinib and 76\% with erlotinib/gefitinib; OR: $1.28 ; 95 \%$ CI: $0.85-1.93 ; p=0.2335$ ). However, median duration of response was 17.2 months with osimertinib versus 8.5 months with erlotinib/gefitinib. At data cutoff, OS data were immature (25\%). Importantly, censoring of OS started at 15 months, which is when crossover from erlotinib/gefitinib to osimertinib may start to have an impact [10]; as such, the OS results are awaited with interest. Similar to the results from AURA and AURA3, plasma testing for EGFR mutations within the FLAURA trial showed good concordance with tissue testing, supporting the use of plasma testing in clinical practice to identify patients suitable for treatment [42].

While FLAURA supports that osimertinib is more effective than erlotinib/gefitinib, the results should be considered further in light of several limitations. First, despite being the only approved second-generation EGFR TKI, afatinib was not included as a comparator. In light of emerging differences between first- and secondgeneration EGFR TKIs observed in LUX-Lung 7 and ARCHER 1050, this precludes any evaluation of clinical benefit for osimertinib over a second-generation EGFR TKI. Additionally, as well as the OS data from FLAURA (and AURA3 for the sequencing strategy), we await further details on efficacy by TKI comparator (erlotinib or gefitinib) and information on subsequent therapies. Based on current standard practice, most patients with EGFR mutation-positive NSCLC receive a first- or second-generation TKI in the first-line until disease progression. Will results from FLAURA change this treatment paradigm? While it would appear likely that osimertinib will become a first-line treatment option for EGFR mutation-positive NSCLC, the optimal first-line choice remains complex. In the next section, we consider some of the key arguments that have emerged following presentation of the FLAURA data.

\section{Factors influencing first-line treatment choice: mechanisms of resistance \& subsequent therapy options}

With the availability of multiple agents for EGFR mutation-positive NSCLC, treatment needs to be considered in terms of a long-term plan to maximize survival. Thus, the sequence of treatment and consideration of the molecular status of the tumor is of key importance. In the Phase III trials of first-generation EGFR TKIs, rates of subsequent therapy were generally high (around 60-70\%) [17,18,20]. With regard to the second-generation EGFR TKIs, detailed analysis of the LUX-Lung 3, 6 and 7 trials showed that $71 \%$ of patients received a further line of treatment following discontinuation of afatinib, with $28 \%$ of patients receiving four lines of subsequent treatment [43]. Subsequent therapy after afatinib predominantly consisted of platinum-based chemotherapy, with others including single-agent chemotherapy, first-generation EGFR TKI monotherapy, chemotherapy combination therapies and third-generation EGFR TKIs, thus suggesting a range of potential treatment options post-afatinib that may impact survival of patients. Furthermore, subsequent treatment duration was similar irrespective of $E G F R$ mutational subgroup (Del19/L858R) [43].

As previously discussed, acquisition of an EGFR T790M mutation is the predominant mechanism of resistance for first- and second-generation TKIs. For patients with T790M-positive NSCLC who have progressed on an EGFR TKI, osimertinib is now a clear treatment of choice based on data from AURA and AURA3. Due to lack of availability of third-generation EGFR TKIs and the fact that T790M testing was not mandated at the time the LUX-Lung 3, 6 and 7 trials were conducted, only a minority of patients $(n=37)$ received osimertinib, primarily in the third- or later-line setting [43]. Analysis of these patients has to be interpreted with caution, based on small sample size and the possibility of sample enrichment; for instance, the median duration of treatment with firstline treatment in these patients was notably long at 21.9 months. Nevertheless, exploratory hypothesis-generating analysis of time on osimertinib and OS in patients starting on afatinib who received subsequent osimertinib in any line was highly encouraging. Median time on osimertinib in any treatment line was 20.2 months, and median follow-up for osimertinib-treated patients was greater than 4 years. At this time, median OS had not yet been reached [43]. In the current treatment landscape, it is likely that many patients will be eligible for, and able to receive 
osimertinib after front-line first- and second-generation EGFR TKIs, with achievement of prolonged OS. However, those data obviously correspond to a focus on patients who ultimately received multiple lines of treatment, and not the whole intent-to-treat population enrolled in those trials.

Of course, we should also consider that patients who receive first- or second-generation EGFR TKIs in first-line can develop resistance based on mechanisms other than T790M acquisition. Other identified mechanisms of acquired resistance include small-cell histologic transformation, MET amplification and HER2 amplification [44]. However, treatment of T790M-negative disease is an area of unmet medical need and, currently, platinum-doublet chemotherapy is the principal treatment option for patients with T790M-negative disease after first- or secondgeneration EGFR TKIs. In some cases, continuation of the first-line EGFR TKI, potentially with local therapy for asymptomatic or loco-regional progression may be feasible. Additionally, the combination of afatinib with the EGFR-targeting monoclonal antibody, cetuximab, was associated with a response rate of $25 \%$ and median PFS of 4.6 months in patients with EGFR TKI-resistant T790M-negative NSCLC [45]. Other options currently under investigation include immuno-oncology agents, such as nivolumab, and novel targeted agents, potentially as combination therapy (e.g., osimertinib plus savolitinib [a MET inhibitor] or selumitinib [a MEK1/2 inhibitor]) [46]. Phase II data with the erlotinib and bevacizumab combination are also available $[47,48]$.

The results from FLAURA pose a challenge for treating physicians. It may seem intuitive to start with an agent that provides improved PFS versus first-generation EGFR TKIs, albeit without any evidence versus second-generation TKIs. However, OS data are immature and, as such, it may be premature to alter standard treatment based on these results. This becomes even more relevant when we consider the potential treatment options post-osimertinib. Resistance mechanisms for osimertinib are currently not well understood and appear to be heterogeneous. Most information available is based on small cohort analyses. For example, in the cohort of treatment-naïve patients in AURA, osimertinib resistance mechanisms were evaluated in 19 patients [49]. Potential resistance mechanisms were identified in nine patients, and included MET, EGFR and KRAS amplification, KRAS or PIK3CA mutations and EGFR C797S mutation. However, resistance mechanisms could not be identified in the remaining ten patients. Another analysis of 23 patients with acquired resistance to osimertinib identified MET amplification and EGFR C797S mutations as the predominant mechanisms of resistance [50]. Other reported mechanisms include small-cell transformation [51-53], EGFR L781Q mutation [54], ERBB2 amplification [55] and BRAF mutations [56]. Preclinical studies have also identified potential resistance mechanisms. For example, cells that are resistant to osimertinib appear to have enhanced levels of phosphorylated MEK and may be particularly sensitive to combined EGFR and MEK inhibition [57,58]. The identification of heterogeneous resistance mechanisms to osimertinib has prompted the initiation of several early-phase clinical trials [59]. The ongoing Phase IB TATTON trial (NCT02143466) is assessing the combination of osimertinib with the MEK1/2 inhibitor, selumetinib, the immune checkpoint inhibitor, durvalumab, or the MET inhibitor, savolitinib, in patients with EGFR mutation-positive NSCLC who have progressed on an EGFR TKI. Other Phase I studies are assessing the combination of osimertinib with the VEGFR2 antibody, ramucirumab, or the EGFR antibody, necitumumab, in patients with T790M-positive NSCLC (NCT02789345) and the combination of osimertinib with navitoclax, a BCL-2 family inhibitor (NCT02520778).

The types of mutations observed with osimertinib have important implications for potential subsequent therapies. For example, C797S mutations can occur in cis (on the same allele) or in trans (on a different allele) with T790M [60] or indeed can occur without T790M (loss of T790M mutation) [61]. For those that occur in trans, combination therapy of first-generation EGFR TKIs with third-generation EGFR TKIs may be feasible [62]. However, C797S mutations in cis with T790M are likely to be resistant to first- and second-generation EGFR TKIs, precluding their use post-osimertinib. Patients with tumors with ERBB2 or MET amplification may be suitable for investigational compounds that target these pathways. However, outside of suitable clinical trials and for unknown driver mutations, the likely second-line treatment after osimertinib will be chemotherapy. In AURA3, subsequent therapy was received in $24 \%$ of patients in the osimertinib group compared with $71 \%$ of the chemotherapy group [9]. In the AURA Phase II expansion cohort, $41 \%$ of patients were treated after progression on osimertinib [7]. While it must be acknowledged that osimertinib was being evaluated in a second-line setting in these trials, these data might indicate limited treatment options after osimertinib.

As such, the overall sequence of first- or second-generation TKIs followed by osimertinib may offer improved survival outcomes versus osimertinib in first-line; OS data from AURA3 and FLAURA will be of interest to analyze treatment sequences further (Figure 3). On the other hand, holding back osimertinib for second-line use will mean that a proportion of patients with EGFR mutation-positive NSCLC will never receive osimertinib and its associated efficacy and tolerability benefits. Nevertheless, given the predominance of T790M-driven resistance $(50-70 \%[6,7]$ 

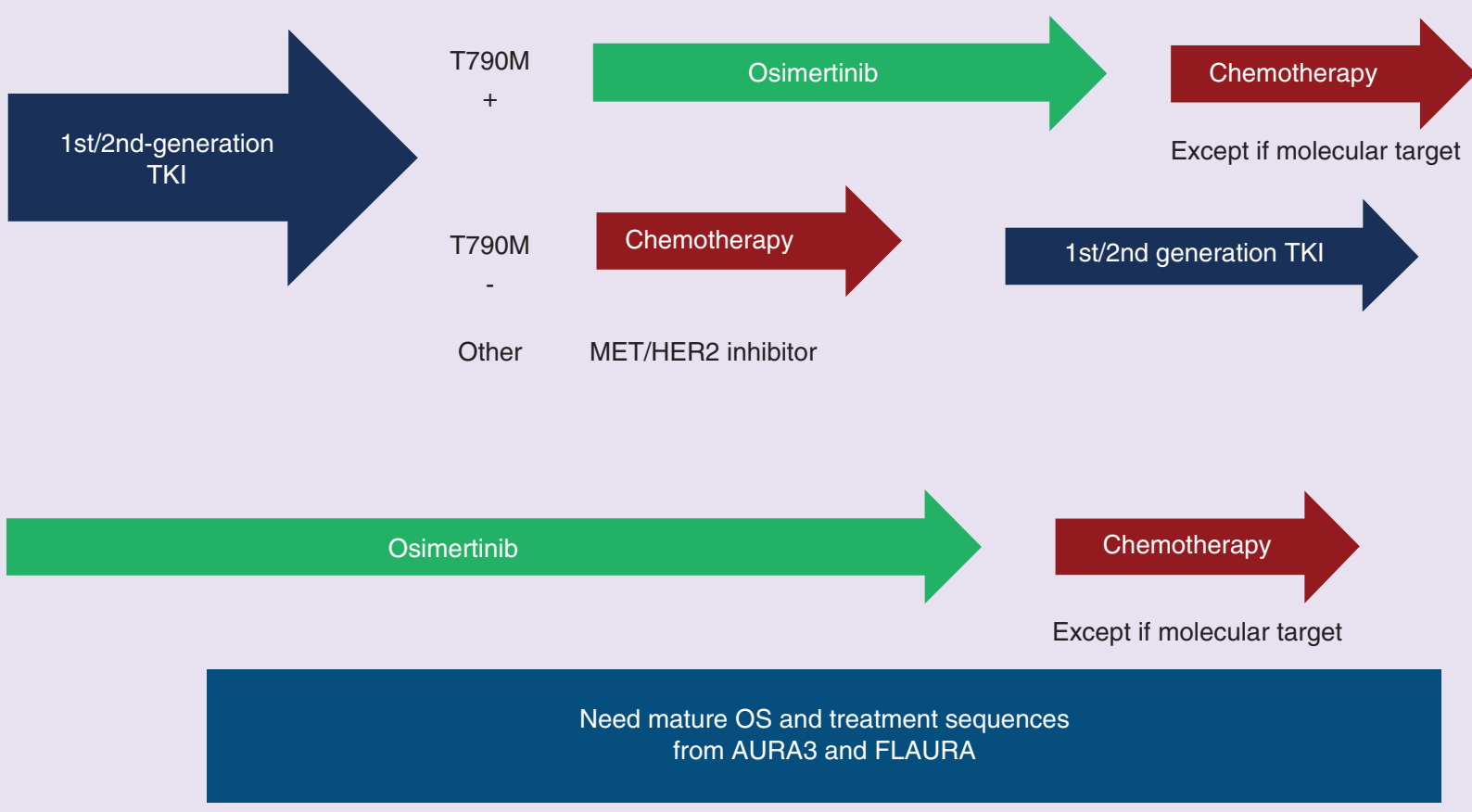

Figure 3. Potential treatment sequences in EGFR mutation-positive non-small-cell lung cancer after first-line EGFR tyrosine kinase inhibitor.

OS: Overall survival; TKI: Tyrosine kinase inhibitor.

of patients) and high uptake of postprogression therapy, around $50 \%$ of patients could ultimately receive sequential EGFR TKIs. Ultimately, prospective clinical trials assessing sequential treatment with first- or second-generation TKIs followed by osimertinib versus osimertinib followed by appropriate second-line treatment are required. The European Organisation for Research and Treatment of Cancer APPLE study is currently ongoing with this aim (NCT02856893), integrating the use of cell-free DNA analyses.

\section{Factors influencing first-line treatment choice: brain metastases}

Patients with EGFR mutation-positive NSCLC appear to be particularly prone to the development of brain metastases [63]. Thus, efficacy and safety of EGFR TKIs in patients with EGFR mutation-positive NSCLC and brain metastases is of high importance. Preclinical studies suggest that osimertinib is able to cross the blood-brain barrier [64]. Moreover, in FLAURA, osimertinib significantly prolonged PFS compared with erlotinib/gefitinib in patients with asymptomatic brain metastases (HR: 0.47; 95\% CI: 0.30-0.74); this difference was a similar magnitude to that in patients without brain metastases (HR: 0.46; 95\% CI: 0.36-0.59). De novo CNS progression events were also lower with osimertinib than erlotinib/gefitinib (6 vs 15\%) [10].

Several prospective studies have indicated that the first-generation TKIs, erlotinib and gefitinib, have clinical activity in patients with NSCLC and brain metastases, although there are limited data regarding direct intracranial activity [65,66]. These findings are consistent with observations that erlotinib and gefitinib can permeate the bloodbrain barrier, albeit inefficiently [67-71]. In an effort to increase drug concentrations in the brain, several studies have assessed high-dose regimens of gefitinib or erlotinib in patients with EGFR mutation-positive NSCLC and brain metastases $[67,69]$. A number of retrospective studies have assessed the combination of first-generation EGFR TKIs with whole-brain radiation therapy (WBRT), which is thought to increase permeability of the bloodbrain barrier [72-74]. These studies indicate that concomitant WBRT may enhance the intracranial activity of first-generation TKIs.

The second-generation inhibitor, afatinib, has demonstrated efficacy in patients with stable brain metastases at baseline. Analysis of the LUX-Lung 3 and 6 trials showed that afatinib improved PFS versus chemotherapy in patients with asymptomatic brain metastases (HR: 0.50; 95\% CI: 0.27-0.95). Afatinib also delayed CNS 
progression compared with chemotherapy, and for most patients with baseline brain metastases, the brain was not the site of first disease progression [75]. Furthermore, an additional competing risk analysis in afatinib-treated patients with baseline brain metastases showed that the cumulative incidence of CNS progression was lower than that of non-CNS progression (31 vs 52\%; Figure 4). Risk of de novo CNS progression with afatinib was also very low (observed in only 6\% of patients receiving afatinib in LUX-Lung 3 and 6). Non-CNS progression for patients without baseline brain metastases was 78\% [Boehringer Ingelheim, Data on File].

Overall, both afatinib and osimertinib have demonstrated activity in patients with asymptomatic brain metastases at standard therapeutic doses, while there are also limited data showing activity of erlotinib and gefitinib in patients with brain metastases, especially as high-dose regimens or in combination with WBRT. Further data on the impact of these agents on brain metastases are required, as well as long-term outcomes (including impact of subsequent therapy), to help determine which agent is the most appropriate first-line option for these patients.

\section{Factors influencing first-line treatment choice: tolerability profile}

EGFR TKIs are associated with a predictable, class-related AE profile, based on their mode of action. Inhibition of wild-type EGFR results in characteristic gastrointestinal (e.g., diarrhea) and skin (e.g., rash) AEs, although there are some differences in the AE profile between different EGFR TKIs. For example, in LUX-Lung 7, the most common drug-related grade $3 / 4$ AEs with afatinib were diarrhea (13\%), rash or acne $(9 \%)$ and fatigue $(6 \%)$, while elevated liver enzymes (9\%) and rash or acne (3\%) were the most common drug-related grade 3/4 AEs with gefitinib [28]. Treatment discontinuations due to treatment-related AEs were equally low in both treatment groups (6.3\%). This is partly due to the well-defined dose adjustment protocol for management of afatinib-associated AEs. Post hoc analysis from LUX-Lung 3, 6 and 7 showed that dose adjustment resulted in a reduction in the frequency and severity of AEs, as would be expected. Importantly, however, this did not impact on efficacy: median PFS was similar in patients who received a dose reduction within the first 6 months of treatment and those who remained on the 40-mg dose (or $50 \mathrm{mg}$ ) [76,77].

Osimertinib and other third-generation EGFR TKIs were designed to be EGFR wild-type sparing in an effort to improve tolerability. The overall incidence of treatment-related AEs in FLAURA was similar between osimertinib and gefitinib (91 and 92\%, respectively), but with a lower incidence of grade $\geq 3$ treatment-related AEs for osimertinib (18 vs 28\%). The most common grade 3/4 treatment-related AEs with osimertinib were decreased appetite $(3 \%)$ and diarrhea (2\%) $[10,78]$. Information on discontinuations due to treatment-related AEs in FLAURA are not available yet; however, the discontinuation rate due to any $\mathrm{AE}$ was $13 \%$ in the osimertinib group and $18 \%$ in the erlotinib/gefitinib group [10].

Overall, osimertinib has a favorable safety profile. However, it should be considered that the most likely secondline treatment after osimertinib will be chemotherapy. The tolerability profile of chemotherapy can be extremely challenging for patients, especially in later-line treatment, with a detrimental impact on patient quality of life. In contrast, the majority of patients receiving a first- or second-generation EGFR TKI will likely be able to receive osimertinib in second-line, thus prolonging their chemotherapy-free period. Given that EGFR TKIs are generally better tolerated than chemotherapy, sequential use of EGFR TKIs may offer improved tolerability and quality of life in the longer term. Again, data from AURA3 and FLAURA are awaited.

One final consideration is the potential for drug-drug interactions. Gefitinib and erlotinib undergo extensive hepatic metabolism via CYP450 enzymes; additionally, erlotinib is a potent inhibitor of CYP1A1 and a moderate inhibitor of CYP3A4 and CYP2C8 [79]. Osimertinib is metabolized by CYP3A4 and CYP3A5, may inhibit breast cancer resistance protein and may induce CYP3A4, CYP1A2 and CYP2C [80,81]. In contrast, afatinib undergoes minimal hepatic metabolism and is not a substrate for CYP-dependent enzymes [79]. This would suggest that afatinib has a low potential for drug-drug interactions, which is an important factor in patients with advanced NSCLC, many of whom may have additional co-morbidities and are likely to be receiving multiple medications.

\section{Conclusion}

Gefitinib, erlotinib and afatinib are approved for the first-line treatment of EGFR mutation-positive NSCLC. Emerging evidence suggests that second-generation inhibitors may be more effective than first-generation TKIs. Following disease progression on these agents, osimertinib, a third-generation EGFR TKI, has shown considerable efficacy in patients with T790M-positive disease, and is approved in this setting. For patients with T790M-negative disease, options are more limited, and chemotherapy is the current principal treatment. Recent data from FLAURA showed that osimertinib had superior PFS to first-generation TKIs in the first-line setting, positioning osimertinib 


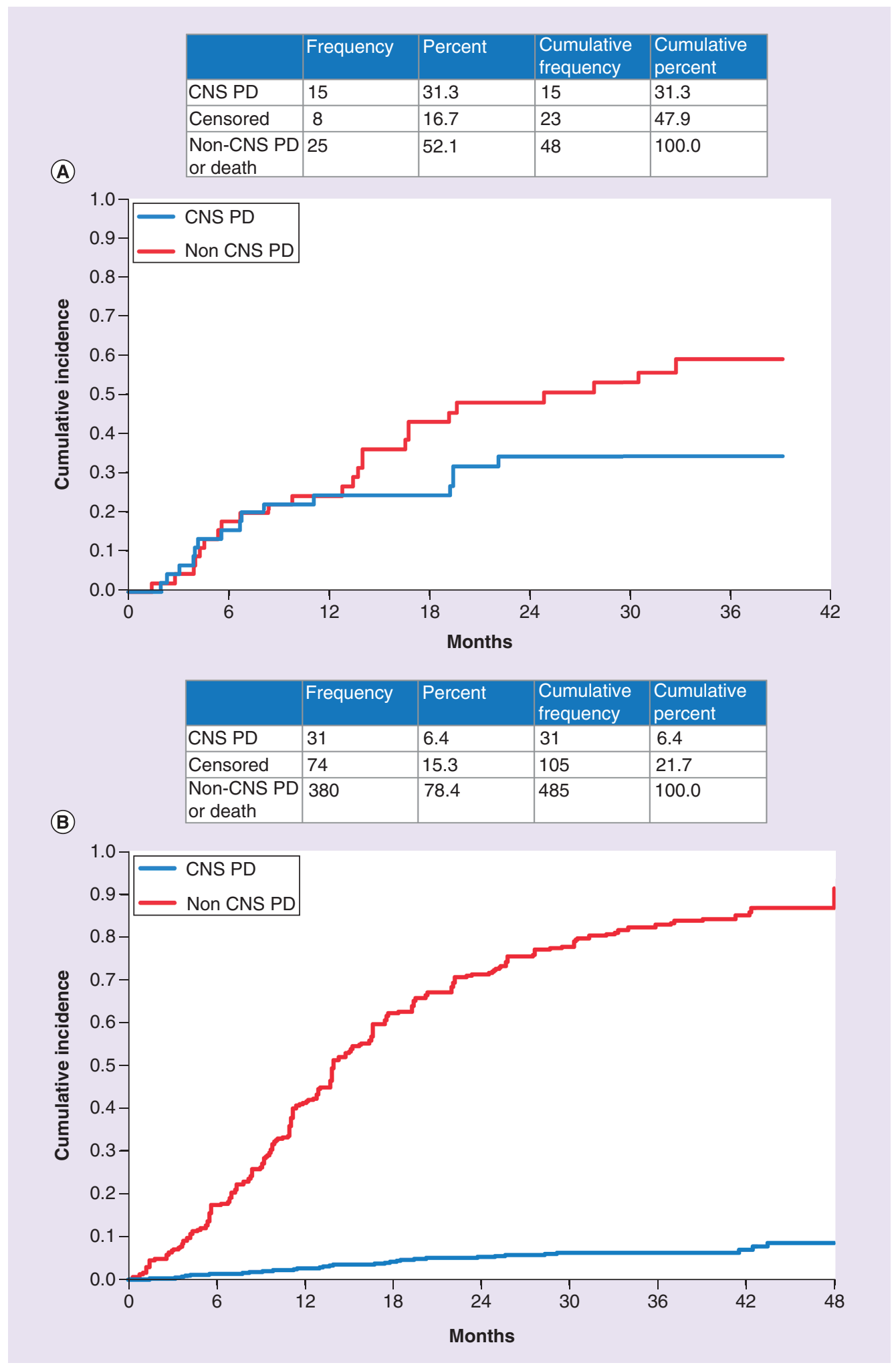

Figure 4. Competing risk analysis for progression in afatinib-treated patients. Risk of progression in patients with baseline brain metastases (LUX-Lung 3 and 6; [A]) and those without baseline brain metastases (LUX-Lung 3, 6 and 7; [ B]).

PD: Progressive disease. 


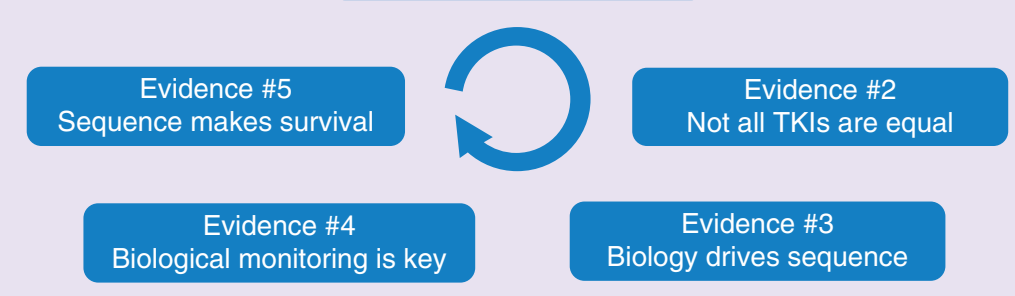

Figure 5. Evidence to evaluate when choosing the sequence in patients with EGFR mutation-positive non-small-cell lung cancer.

TKI: Tyrosine kinase inhibitor.

as another first-line choice. While, given the results from FLAURA, it may seem intuitive to use osimertinib in the first-line, it is important to consider the long-term treatment plan. Currently, subsequent treatment options for osimertinib are not clearly defined, and mature OS data are not available. Extent of OS from FLAURA will be of great interest, as we cannot extrapolate from the sum of median PFS in individual trials. In LUX-Lung 3, 6 and 7, afatinib was associated with median OS of around 30 months in patients with NSCLC and common (Del19/L858R) mutations, despite few patients receiving a subsequent T790M inhibitor (due to availability) [26,82]. In some cases, it may also be appropriate for patients to receive treatment beyond progression (as seen in LUX-Lung 7 and AURA3). In addition, use of local therapies with continued EGFR TKI treatment, for oligoprogression or focal progression, may provide long-term disease control for some patients [83].

Although there is a lack of data-assessing sequential treatment with EGFR TKIs, initial hypothesis-generating data show that sequential afatinib followed by osimertinib confers encouraging survival outcomes in post hoc analysis (median OS not yet reached after a median follow-up of $>4$ years) for the subset of patients treated with the sequence. Overall, further research is needed to determine the most appropriate sequential therapy to optimize clinical benefit in patients with EGFR mutation-positive NSCLC. An illustrated summary of this section can be found in Figure 5.

\section{Future perspective}

Over the next 5-10 years, it is likely that research will focus on outcomes and tolerability of different sequences, for example, first- or second-generation TKIs followed by a third-generation TKI, or a third-generation TKI followed by appropriate treatment. This will enable us to determine the optimal treatment sequence to prolong patient survival, while maintaining quality of life. Additional research on mechanisms of resistance and suitable posttreatment options for the third-generation TKI, osimertinib, will be of great interest, particularly if it is possible to target specific mutations and thus enable personalized medicine. There is rationale for novel combination regimens in the post-osimertinib setting and the results of ongoing early-phase trials are eagerly awaited. Treatment options for patients with T790M-negative disease following progression on an EGFR TKI are also likely to evolve, given the unmet medical need in this patient population. Further assessment of currently available EGFR TKIs for patients with brain metastases could help to define the optimal treatment for these patients, along with novel agents that can effectively penetrate the blood-brain barrier.

\section{Supplementary data}

A video abstract and transcript are available as an accompaniment to this paper. To view the supplementary transcript for this please visit the journal website at: www.futuremedicine.com/doi/suppl/10.2217/fon-2017-0636.

Financial \& competing interests disclosure

N Girard has acted as a consultant and received financial assistance for travel from AstraZeneca, Hoffman LaRoche, Boehringer Ingelheim and Pfizer. The author has also received academic grants from AstraZeneca, Hoffman LaRoche, Boehringer Ingelheim and Pfizer. The author has no other relevant affiliations or financial involvement with any organization or entity with a financial interest in or financial conflict with the subject matter or materials discussed in the manuscript apart from those disclosed. 
Medical writing assistance, supported financially by Boehringer Ingelheim, was provided by C Allinson of GeoMed, an Ashfield company, part of UDG Healthcare plc, during the preparation of this article.

Open access

This work is licensed under the Attribution-NonCommercial-NoDerivatives 4.0 Unported License. To view a copy of this license, visit http://creativecommons.org/licenses/by-nc-nd/4.0/.

\section{Executive summary}

First- \& second-generation EGFR tyrosine kinase inhibitors

- The first- and second-generation EGFR tyrosine kinase inhibitors (TKIs), erlotinib, gefitinib and afatinib, have demonstrated robust efficacy for the first-line treatment of patients with EGFR mutation-positive non-small-cell lung cancer (NSCLC), and are approved in this indication.

- Recent data suggest that second-generation ERBB family blockers, afatinib and dacomitinib, have improved efficacy compared with first-generation EGFR TKIs, erlotinib and gefitinib.

Third-generation EGFR TKIs in patients with acquired resistance

- Despite the evidence observed in the first-line setting with first- and second-generation TKIs, resistance develops, predominantly due to acquisition of the EGFR T790M mutation.

- The third-generation EGFR TKIs show high potency for T790M mutations. Osimertinib significantly prolonged progression-free survival versus chemotherapy in patients with T790M-positive NSCLC who had disease progression after first-line EGFR TKI therapy, and is approved in this setting.

Third-generation EGFR TKIs as first-line treatment

- Recently, osimertinib has demonstrated improved progression-free survival versus the first-generation EGFR TKIs in the first-line setting.

Factors influencing first-line treatment choice: mechanisms of resistance \& subsequent therapy

- For patients who receive a first- or second-generation EGFR TKI and experience resistance due to a T790M mutation (around $50-70 \%$ of cases), osimertinib is a clear treatment of choice.

- For patients who receive a first- or second-generation EGFR TKI and experience resistance due to another mechanism, chemotherapy is the principal treatment; options, including afatinib in combination with cetuximab or immuno-oncology agents in combination with novel targeted agents, are under investigation.

- Resistance mechanisms for osimertinib appear heterogeneous, with MET amplification, small-cell transformation and EGFR C797S mutation identified as the main mechanisms. The likely current second-line treatment option post-osimertinib will be chemotherapy, although investigational agents within clinical trials may be suitable for particular mutations.

- Further information on the overall sequence of first- or second-generation TKIs followed by osimertinib versus osimertinib in first-line are required.

Factors influencing first-line treatment choice: brain metastases

- Osimertinib and afatinib have both demonstrated activity in patients with brain metastases in prospective randomized trials, while evidence for erlotinib and gefitinib is more limited.

- Further information on long-term outcomes of patients with brain metastases is required to determine the best first-line treatment option.

Factors influencing first-line treatment choice: tolerability profile

- EGFR TKIs have a predictable, class-related adverse event (AE) profile, although there are some differences between different EGFR TKIs.

- Afatinib has a well-defined dose adjustment protocol for management of treatment-related AEs, which does not impact on efficacy.

- Osimertinib, which is EGFR wild-type sparing, has a favorable safety profile, with a lower incidence of grade $\geq 3$ treatment-related AEs than gefitinib.

- The tolerability of the overall treatment sequence should also be considered.

Conclusion

- Erlotinib, gefitinib and afatinib are approved for the first-line treatment of EGFR mutation-positive NSCLC, and recent data have also positioned osimertinib as a potential first-line treatment option.

- Selecting the optimal first-line treatment requires consideration of patient factors, subsequent therapy options for long-term treatment and the tolerability profile of the overall treatment sequence. 


\section{References}

Papers of special note have been highlighted as: $\bullet$ of interest; $\bullet \bullet$ of considerable interest

1. Chan BA, Hughes BG. Targeted therapy for non-small cell lung cancer: current standards and the promise of the future. Transl. Lung Cancer Res. 4(1), 36-54 (2015).

2. Torti $\mathrm{D}$, Trusolino L. Oncogene addiction as a foundational rationale for targeted anti-cancer therapy: promises and perils. EMBO Mol. Med. 3(11), 623-636 (2011).

3. Marshall J. Clinical implications of the mechanism of epidermal growth factor receptor inhibitors. Cancer 107(6), 1207-1218 (2006).

4. Novello S, Barlesi F, Califano R et al. Metastatic non-small-cell lung cancer: ESMO Clinical Practice Guidelines for diagnosis, treatment and follow-up. Ann. Oncol. 27(Suppl. 5), v1-v27 (2016).

5. Solca F, Dahl G, Zoephel A et al. Target binding properties and cellular activity of afatinib (BIBW 2992), an irreversible ErbB family blocker. J. Pharmacol. Exp. Ther. 343(2), 342-350 (2012).

6. Sequist LV, Waltman BA, Dias-Santagata $\mathrm{D}$ et al. Genotypic and histological evolution of lung cancers acquiring resistance to EGFR inhibitors. Sci. Transl. Med. 3(75), 75 ra26 (2011).

7. Yang JC, Ahn MJ, Kim DW et al. Osimertinib in pretreated T790M-positive advanced non-small-cell lung cancer: AURA study Phase II extension component. J. Clin. Oncol. 35(12), 1288-1296 (2017).

-. AURA Phase II extension, showing the presence of T790M mutation in up to $70 \%$ of patients who had experienced disease progression on first-line afatinib, erlotinib or gefitinib.

8. Janne PA, Yang JC, Kim DW et al. AZD9291 in EGFR inhibitor-resistant non-small-cell lung cancer. N. Engl. J. Med. 372(18), 1689-1699 (2015).

9. Mok TS, Wu YL, Ahn MJ et al. Osimertinib or platinum-pemetrexed in EGFR T790M-positive lung cancer. N. Engl. J. Med. 376(7), 629-640 (2017).

-. AURA3 randomized trial, which showed that osimertinib prolonged progression-free survival (PFS) compared with chemotherapy in patients with T790M-positive non-small-cell lung cancer (NSCLC) who had experienced disease progression during first-line EGFR tyrosine kinase inhibitor (TKI) therapy.

10. Soria JC, Ohe Y, Vansteenkiste J et al. Osimertinib in untreated EGFR-mutated advanced non-small-cell lung cancer. N. Engl. J. Med. 378, 113-125 (2018).

-. First presentation of the FLAURA data, which showed that osimertinib, a third-generation TKI, demonstrated improved PFS compared with the first-generation TKIs, erlotinib or gefitinib, in the first-line setting.

11. Zhou C, Wu YL, Chen G et al. Erlotinib versus chemotherapy as first-line treatment for patients with advanced EGFR mutation-positive non-small-cell lung cancer (OPTIMAL, CTONG-0802): a multicentre, open-label, randomised, Phase III study. Lancet Oncol. 12(8), 735-742 (2011).

12. Rosell R, Carcereny E, Gervais R et al. Erlotinib versus standard chemotherapy as first-line treatment for European patients with advanced EGFR mutation-positive non-small-cell lung cancer (EURTAC): a multicentre, open-label, randomised Phase III trial. Lancet Oncol. 13(3), 239-246 (2012).

13. Wu YL, Zhou C, Liam CK et al. First-line erlotinib versus gemcitabine/cisplatin in patients with advanced EGFR mutation-positive non-small-cell lung cancer: analyses from the Phase III, randomized, open-label, ENSURE study. Ann. Oncol. 26(9), 1883-1889 (2015).

14. Mok TS, Wu YL, Thongprasert S et al. Gefitinib or carboplatin-paclitaxel in pulmonary adenocarcinoma. N. Engl. J. Med. 361(10), 947-957 (2009).

15. Mitsudomi T, Morita S, Yatabe Y et al. Gefitinib versus cisplatin plus docetaxel in patients with non-small-cell lung cancer harbouring mutations of the epidermal growth factor receptor (WJTOG3405): an open label, randomised Phase III trial. Lancet Oncol. 11(2), 121-128 (2010).

16. Maemondo M, Inoue A, Kobayashi K et al. Gefitinib or chemotherapy for non-small-cell lung cancer with mutated EGFR. N. Engl. J. Med. 362(25), 2380-2388 (2010).

17. Fukuoka M, Wu YL, Thongprasert $S$ et al. Biomarker analyses and final overall survival results from a Phase III, randomized, open-label, first-line study of gefitinib versus carboplatin/paclitaxel in clinically selected patients with advanced non-small-cell lung cancer in Asia (IPASS). J. Clin. Oncol. 29(21), 2866-2874 (2011).

18. Inoue $\mathrm{A}$, Kobayashi $\mathrm{K}$, Maemondo $\mathrm{M}$ et al. Updated overall survival results from a randomized Phase III trial comparing gefitinib with carboplatin-paclitaxel for chemo-naive non-small cell lung cancer with sensitive EGFR gene mutations (NEJ002). Ann. Oncol. 24(1), 54-59 (2013).

19. Yoshioka H, Mitsudomi T, Morita S et al. Final overall survival results of WJTOG 3405, a randomized Phase III trial comparing gefitinib (G) with cisplatin plus docetaxel (CD) as the first-line treatment for patients with non-small cell lung cancer (NSCLC) harboring mutations of the epidermal growth factor receptor (EGFR). J. Clin. Oncol. 32(Suppl. 15), 8117 (2014).

20. Zhou C, Wu YL, Chen G et al. Final overall survival results from a randomised, Phase III study of erlotinib versus chemotherapy as first-line treatment of EGFR mutation-positive advanced non-small-cell lung cancer (OPTIMAL, CTONG-0802). Ann. Oncol. 26(9), 1877-1883 (2015). 
21. Leon LF, Golsorkhi A, Liu S, Al E. Overall survival analyses of first-line erlotinib versus chemotherapy in the EURTAC study population controlling for the use of post-study therapy. Annal. Oncol. 25, iv426-iv470 (2014).

22. Yang J, Wu YL, Saijo S, Al. E. Efficacy outcomes in first-line treatment of advanced NSCLC with gefitinib (G) vs carboplatin/paclitaxel (C/P) by epidermal growth factor receptor (EGFR) gene-copy number score and by most common EGFR mutation subtypes exploratory data from IPASS. Eur. J. Cancer 47, S633 (2011).

23. Watanabe S, Inoue A, Nukiwa T, Kobayashi K. Comparison of gefitinib versus chemotherapy in patients with non-small cell lung cancer with exon 19 deletion. Anticancer Res. 35(12), 6957-6961 (2015).

24. Mitsudomi T, Morita S, Yatabe Y et al. Updated overall survival results of WJTOG 3405, a randomized Phase III trial comparing gefitinib $(\mathrm{G})$ with cisplatin plus docetaxel $(\mathrm{CD})$ as the first-line treatment for patients with non-small cell lung cancer harboring mutations of the epidermal growth factor receptor (EGFR). J. Clin. Oncol. 30(Suppl. 15), abstract 7521 (2012).

25. Sequist LV, Yang JC, Yamamoto $\mathrm{N}$ et al. Phase III study of afatinib or cisplatin plus pemetrexed in patients with metastatic lung adenocarcinoma with EGFR mutations. J. Clin. Oncol. 31(27), 3327-3334 (2013).

26. Yang JC, Wu YL, Schuler M et al. Afatinib versus cisplatin-based chemotherapy for EGFR mutation-positive lung adenocarcinoma (LUX-Lung 3 and LUX-Lung 6): analysis of overall survival data from two randomised, Phase III trials. Lancet Oncol. 16(2), 141-151 (2015).

27. Wu YL, Zhou C, Hu CP et al. Afatinib versus cisplatin plus gemcitabine for first-line treatment of Asian patients with advanced non-small-cell lung cancer harbouring EGFR mutations (LUX-Lung 6): an open-label, randomised Phase III trial. Lancet Oncol. 15(2), 213-222 (2014).

28. Park K, Tan EH, O’Byrne K et al. Afatinib versus gefitinib as first-line treatment of patients with EGFR mutation-positive non-small-cell lung cancer (LUX-Lung 7): a Phase IIB, open-label, randomised controlled trial. Lancet Oncol. 17(5), 577-589 (2016).

-• Primary analysis of the LUX-Lung 7 trial, the first head-to-head trial comparing a first-generation TKI (gefitinib) with a second-generation ERBB family blocker (afatinib) as first-line treatment of EGFR mutation-positive NSCLC and illustrating improved PFS with afatinib.

29. Paz-Ares L, Tan EH, O’byrne K et al. Afatinib versus gefitinib in patients with EGFR mutation-positive advanced non-small-cell lung cancer: overall survival data from the Phase IIb LUX-Lung 7 trial. Ann. Oncol. 28(2), 270-277 (2017).

30. Wu YL, Cheng Y, Zhou X et al. Dacomitinib versus gefitinib as first-line treatment for patients with EGFR-mutation-positive non-small-cell lung cancer (ARCHER 1050): a randomised, open-label, Phase III trial. Lancet Oncol. 18 (11 ), 1454 -1466 (2017).

31. Jackman DM, Yeap BY, Sequist LV et al. Exon 19 deletion mutations of epidermal growth factor receptor are associated with prolonged survival in non-small cell lung cancer patients treated with gefitinib or erlotinib. Clin. Cancer Res. 12(13), 3908-3914 (2006).

32. Riely GJ, Pao W, Pham D et al. Clinical course of patients with non-small cell lung cancer and epidermal growth factor receptor exon 19 and exon 21 mutations treated with gefitinib or erlotinib. Clin. Cancer Res. 12, 839-844 (2006).

33. Yang JC, Hirsh V, Schuler M et al. Symptom control and quality of life in LUX-Lung 3: a Phase III study of afatinib or cisplatin/pemetrexed in patients with advanced lung adenocarcinoma with EGFR mutations. J. Clin. Oncol. 31(27), 3342-3350 (2013).

34. Wu Y-L, Cheng Y, Zhou X et al. Dacomitinib versus gefitinib as first-line treatment for patients with EGFR-mutation-positive non-small-cell lung cancer (ARCHER 1050): a randomised, open-label, Phase III trial. Lancet Oncol. 18(11), 1454-1466 (2017).

- Phase III ARCHER 1050 study showing that the second-generation ERBB family blocker, dacomitinib, was more effective than the first-generation TKI, gefitinib, as first-line treatment of EGFR mutation-positive NSCLC.

35. Cross DA, Ashton SE, Ghiorghiu S et al. AZD9291, an irreversible EGFR TKI, overcomes T790M-mediated resistance to EGFR inhibitors in lung cancer. Cancer Discov. 4(9), 1046-1061 (2014).

36. Li D, Ambrogio L, Shimamura T et al. BIBW2992, an irreversible EGFR/HER2 inhibitor highly effective in preclinical lung cancer models. Oncogene 27(34), 4702-4711 (2008).

37. Goss G, Tsai CM, Shepherd FA et al. Osimertinib for pretreated EGFR Thr790Met-positive advanced non-small-cell lung cancer (AURA2): a multicentre, open-label, single-arm, Phase II study. Lancet Oncol. 17(12), 1643-1652 (2016).

38. Mitsudomi T, Ahn M-J, Bazhenova L et al. Overall survival (OS) in patients (pts) with EGFR T790M-positive advanced non-small cell lung cancer (NSCLC) treated with osimertinib: results from two Phase II studies. Ann. Oncol. 28(Suppl. 5), v460-v496 (2017).

39. Huang WL, Chen YL, Yang SC et al. Liquid biopsy genotyping in lung cancer: ready for clinical utility? Oncotarget 8(11), 18590-18608 (2017).

40. Oxnard GR, Thress KS, Alden RS et al. Association between plasma genotyping and outcomes of treatment with osimertinib (AZD9291) in advanced non-small-cell lung cancer. J. Clin. Oncol. 34(28), 3375-3382 (2016).

41. Ahn M, Han J, Tsai C et al. Detection of EGFR mutations from plasma ctDNA in the osimertinib Phase III trial (AURA3): comparison of three plasma assays. Presented at: IASLC 18th World Conference on Lung Cancer (WCLC). Yokohama, Japan, 15-18 October (2017).

42. Gray J, Okamoto I, Sriuranpong V et al. Osimertinib vs SoC EGFR TKI as first-line treatment in patients with EGFRm advanced NSCLC (FLAURA): plasma ctDNA analysis. Presented at: IASLC 18th World Conference on Lung Cancer (WCLC). Yokohama, Japan, 15-18 October (2017). 
43. Sequist $\mathrm{L}$, Wu Y, Schuler M et al. Subsequent therapies post-afatinib among patients with EGFR mutation-positive NSCLC in LUX-Lung (LL) 3, 6 and 7. Ann. Oncol. 28(Suppl. 5), v460-v496 (2017).

-• Retrospective analysis of subsequent therapy post-afatinib in patients with EGFR mutation-positive (Del19/L858R) NSCLC, illustrating high rates of subsequent therapy and highly encouraging overall survival data for thesequence of afatinib followed by osimertinib.

44. Yu HA, Arcila ME, Rekhtman N et al. Analysis of tumor specimens at the time of acquired resistance to EGFR-TKI therapy in 155 patients with EGFR-mutant lung cancers. Clin. Cancer Res. 19(8), 2240-2247 (2013).

45. Janjigian YY, Smit EF, Groen HJ et al. Dual inhibition of EGFR with afatinib and cetuximab in kinase inhibitor-resistant EGFR-mutant lung cancer with and without T790M mutations. Cancer Discov. 4(9), 1036-1045 (2014).

46. Corallo S, D’Argento E, Strippoli A et al. Treatment options for EGFR T790M-negative EGFR tyrosine kinase inhibitor-resistant non-small cell lung cancer. Target Oncol. 12, 153-161 (2017).

47. Rosell R, Dafni U, Felip E et al. Erlotinib and bevacizumab in patients with advanced non-small-cell lung cancer and activating EGFR mutations (BELIEF): an international, multicentre, single-arm, Phase II trial. Lancet Respir. Med. 5(5), 435-444 (2017).

48. Seto T, Kato T, Nishio M et al. Erlotinib alone or with bevacizumab as first-line therapy in patients with advanced non-squamous non-small-cell lung cancer harbouring EGFR mutations (JO25567): an open-label, randomised, multicentre, Phase II study. Lancet Oncol. 15(11), 1236-1244 (2014).

49. Ramalingam SS, Yang JC, Lee CK et al. Osimertinib as first-line treatment of EGFR mutation-positive advanced non-small-cell lung cancer. J. Clin. Oncol. 36(9), 841-849 (2018).

50. Piotrowska Z, Thress KS, Mooradian M et al. MET amplification (amp) as a resistance mechanism to osimertinib. J. Clin. Oncol. 35 , abstract 9020 (2017).

51. Kim TM, Song A, Kim DW et al. Mechanisms of acquired resistance to AZD9291: a mutation-selective, irreversible EGFR inhibitor. J. Thorac. Oncol. 10(12), 1736-1744 (2015).

52. Li L, Wang H, Li C, Wang Z, Zhang P, Yan X. Transformation to small-cell carcinoma as an acquired resistance mechanism to AZD9291: a case report. Oncotarget 8(11), 18609-18614 (2017).

53. Yang JC, Yu C, Shih J et al. Genomic analysis of tumor and plasma in T790M mutant positive EGFR lung cancer patients before and after osimertinib treatment. Presented at: IASLC 18th World Conference on Lung Cancer (WCLC). Yokohama, Japan, 15-18 October (2017).

54. Bersanelli M, Minari R, Bordi P et al. L718Q mutation as new mechanism of acquired resistance to AZD9291 in EGFR-mutated NSCLC. J. Thorac. Oncol. 11(10), e121-123 (2016).

55. Planchard D, Loriot Y, Andre F et al. EGFR-independent mechanisms of acquired resistance to AZD9291 in EGFR T790M-positive NSCLC patients. Ann. Oncol. 26(10), 2073-2078 (2015).

56. Ho CC, Liao WY, Lin CA, Shih JY, Yu CJ, Chih-Hsin Yang J. Acquired BRAF V600E mutation as resistant mechanism after treatment with osimertinib. J. Thorac. Oncol. 12(3), 567-572 (2017).

57. Eberlein CA, Stetson D, Markovets AA et al. Acquired resistance to the mutant-selective EGFR inhibitor AZD9291 is associated with increased dependence on RAS signaling in preclinical models. Cancer Res. 75(12), 2489-2500 (2015).

58. Ortiz-Cuaran S, Scheffler M, Plenker D et al. Heterogeneous mechanisms of primary and acquired resistance to third-generation EGFR inhibitors. Clin. Cancer Res. 22(19), 4837-4847 (2016).

59. Santarpia M, Liguori A, Karachaliou N et al. Osimertinib in the treatment of non-small-cell lung cancer: design, development and place in therapy. Lung Cancer (Auckl.) 8, 109-125 (2017).

60. Niederst MJ, Hu H, Mulvey HE et al. The allelic context of the C797S mutation acquired upon treatment with third-generation EGFR inhibitors impacts sensitivity to subsequent treatment strategies. Clin. Cancer Res. 21(17), 3924-3933 (2015).

61. Thress KS, Paweletz CP, Felip E et al. Acquired EGFR C797S mutation mediates resistance to AZD9291 in non-small cell lung cancer harboring EGFR T790M. Nat. Med. 21(6), 560-562 (2015).

62. Wang Z, Yang JJ, Huang J et al. Lung adenocarcinoma harboring EGFR T790M and in trans C797S responds to combination therapy of first- and third-generation EGFR TKIs and shifts allelic configuration at resistance. J. Thorac. Oncol. 12(11), 1723-1727 (2017).

63. Hendriks LE, Smit EF, Vosse BA et al. EGFR mutated non-small cell lung cancer patients: more prone to development of bone and brain metastases? Lung Cancer 84(1), 86-91 (2014).

64. Ballard P, Yates JW, Yang Z et al. Preclinical comparison of osimertinib with other EGFR-TKIs in EGFR-mutant NSCLC brain metastases models, and early evidence of clinical brain metastases activity. Clin. Cancer Res. 22(20), 5130-5140 (2016).

65. Iuchi T, Shingyoji M, Sakaida T et al. Phase II trial of gefitinib alone without radiation therapy for Japanese patients with brain metastases from EGFR-mutant lung adenocarcinoma. Lung Cancer 82(2), 282-287 (2013).

66. Park SJ, Kim HT, Lee DH et al. Efficacy of epidermal growth factor receptor tyrosine kinase inhibitors for brain metastasis in non-small cell lung cancer patients harboring either exon 19 or 21 mutation. Lung Cancer 77(3), 556-560 (2012). 
67. Clarke JL, Pao W, Wu N, Miller VA, Lassman AB. High dose weekly erlotinib achieves therapeutic concentrations in CSF and is effective in leptomeningeal metastases from epidermal growth factor receptor mutant lung cancer. J. Neurooncol. 99(2), 283-286 (2010).

68. Deng Y, Feng W, Wu J et al. The concentration of erlotinib in the cerebrospinal fluid of patients with brain metastasis from non-small-cell lung cancer. Mol. Clin. Oncol. 2(1), 116-120 (2014).

69. Hata A, Kaji R, Fujita S, Katakami N. High-dose erlotinib for refractory brain metastases in a patient with relapsed non-small cell lung cancer. J. Thorac. Oncol. 6(3), 653-654 (2011).

70. Lee E, Keam B, Kim DW et al. Erlotinib versus gefitinib for control of leptomeningeal carcinomatosis in non-small-cell lung cancer. $J$. Thorac. Oncol. 8(8), 1069-1074 (2013).

71. Togashi Y, Masago K, Masuda S et al. Cerebrospinal fluid concentration of gefitinib and erlotinib in patients with non-small cell lung cancer. Cancer Chemother. Pharmacol. 70(3), 399-405 (2012).

72. Zhuang H, Yuan Z, Wang J, Zhao L, Pang Q, Wang P. Phase II study of whole brain radiotherapy with or without erlotinib in patients with multiple brain metastases from lung adenocarcinoma. Drug Des. Devel. Ther. 7, 1179-1186 (2013).

73. Zeng YD, Zhang L, Liao $\mathrm{H}$ et al. Gefitinib alone or with concomitant whole brain radiotherapy for patients with brain metastasis from non-small-cell lung cancer: a retrospective study. Asian Pac. J. Cancer Prev. 13(3), 909-914 (2012).

74. Zhu Q, Sun Y, Cui Y et al. Clinical outcome of tyrosine kinase inhibitors alone or combined with radiotherapy for brain metastases from epidermal growth factor receptor (EGFR) mutant non small cell lung cancer (NSCLC). Oncotarget 8(8), 13304-13311 (2017).

75. Schuler M, Wu YL, Hirsh V et al. First-line afatinib versus chemotherapy in patients with non-small cell lung cancer and common epidermal growth factor receptor gene mutations and brain metastases. J. Thorac. Oncol. 11(3), 380-390 (2016).

- Prespecified subgroup analysis of LUX-Lung 3 and 6 trials that showed afatinib prolonged PFS versus chemotherapy in patients with EGFR mutation-positive NSCLC and asymptomatic brain metastases.

76. Hirsh V, Yang JC-H, Tan EH et al. First-line afatinib versus gefitinib for patients with EGFR mutation-positive NSCLC (LUX-Lung 7): patient-reported outcomes and impact of dose modifications on efficacy and adverse events. J. Clin. Oncol. 34(Suppl. 15), abstract 9046 (2016).

77. Yang JC, Sequist LV, Zhou C et al. Effect of dose adjustment on the safety and efficacy of afatinib for EGFR mutation-positive lung adenocarcinoma: post hoc analyses of the randomized LUX-Lung 3 and 6 trials. Ann. Oncol. 27(11), 2103-2110 (2016).

- Post hoc analysis of the LUX-Lung 3 and 6 trials showing that tolerability-guided dose adjustment reduced the frequency and intensity of adverse events associated with afatinib without compromising efficacy.

78. Ramalingam S, Reungwetwattana T, Chewaskulyong B et al. Osimertinib vs standard of care (SoC) EGFR-TKI as first-line therapy in patients (pts) with EGFRm advanced NSCLC: FLAURA. Ann. Oncol. 28(Suppl. 5), v605-v649 (2017).

79. Peters S, Zimmermann S, Adjei AA. Oral epidermal growth factor receptor tyrosine kinase inhibitors for the treatment of non-small cell lung cancer: comparative pharmacokinetics and drug-drug interactions. Cancer Treat. Rev. 40(8), 917-926 (2014).

80. AstraZeneca. TAGRISSO product monograph. (2016). www.astrazeneca.ca/content/dam/az-ca/downloads/productinformation/TAGRISSO\%20-\%20Product-Monograph.pdf

81. European Medicines Agency. TAGRISSO summary of product characteristics. (2016). www.ema.europa.eu/docs/en_GB/document_library/EPAR_-_Product_Information/human/004124/WC500202022.pdf

82. Corral J, Park K, Yang JC-H et al. Afatinib versus gefitinib in patients with EGFR mutation-positive (EGFRm+) NSCLC: updated overall survival data from the Phase IIb trial LUX-Lung 7. Ann. Oncol. 28(Suppl. 2), ii28-ii51 (2017).

83. Conforti F, Catania C, Toffalorio F et al. EGFR tyrosine kinase inhibitors beyond focal progression obtain a prolonged disease control in patients with advanced adenocarcinoma of the lung. Lung Cancer 81(3), 440-444 (2013). 